\title{
The Quality of Her-2/Neu Predictive Immunohistochemistry: Something FISHy?
}

The American Cancer Society estimates that approximately 175,000 women will be identified in 1999 with new cases of breast cancer and approximately 43,000 will die of the disease (1). An increasing number of these cases are diagnosed at an early stage when there is no evidence of lymph node metastases. Tumor biology differs substantially among cases in such low-stage tumors, and it has become increasingly important to identify ancillary tools which allow the pathologist to reliably identify (1) Harbingers of aggressive biologic potential (early recurrence, metastases, death) and/or (2) genotypic or phenotypic features that can be linked to specific therapy based on blocking receptors or specific agents targeted to kill tumor cells.

The exact role for ancillary studies in the diagnosis and management of breast carcinoma remains controversial, even though many of these tools have been available and in use for over a decade. A national consensus does not exist as to which, if any, ancillary studies should be measured in newly diagnosed patients with breast carcinoma. There are certainly many reasons for uncertainty and lack of consensus. Chief among them are the plethora of univariate and poorly controlled literature reports of the correlation of ancillary studies with recurrence and other hallmarks of biologic behavior. A careful review of this literature is confusing at a minimum and generally depressing. For example, "breast cancer" is used not infrequently as an admission criterion to the study, with no exclusion of in situ (as opposed to invasive) ductal carcinoma, exclusion of lymphoma, sarcoma, or specific morphologic variants of invasive ductal breast carcinoma. Variation in methods (sampling, fixation, cell conditioning, instrumentation, reagent sources and stability, interpretation, and others) may also contribute to the confusion and lack of consensus.

Her-2/neu (c-erb-B2) is an oncogene related to the epidermal growth factor receptor family. It is overexpressed on a subset of in situ and invasive carcinomas of the breast. Genomic amplification of the Her-2/neu gene in patients with invasive breast carcinoma has been associated with a significant reduction in metastasis-free survival; similarly staged patients without Her-2/neu genomic amplification have a very high likelihood of remaining cancer free years after removal of the tumor (2-10). With the advent of a humanized monoclonal antibody to the encoded protein on the surface of mammary carcinoma tumor cells, the laboratory assessment of Her-2/neu status has assumed even more importance (11-14). As a predictive factor, Her-2 amplification/overexpression has been found to be related to benefit from adjuvant doxorubicin $(15,16)$ and lack of benefit from adjuvant tamoxifen (17) and CMF (18).

In the current issue of Modern Pathology, the controversial relationship between results derived from fluorescence in situ hybridization (FISH) and immunohistochemical detection of encoded protein is addressed (19). The authors utilized whole cell preparations for FISH rather than paraffin sections, the more standard approach for FISH Her-2/ neu detection. The inability to distinguish between cells derived from in situ rather than invasive carcinoma could limit the practical application of such an approach. High levels of gene amplification were associated with strong membrane immunostaining. Gene copy greater than four and less than 10 was usually associated with chromosome 17 polysomy. The authors used two-color FISH to simultaneously assess Her-2/ neu and chromosome 17 ploidy; given the low frequency of intermediate level Her-2/ neu gene amplification, sequential single-color FISH for such cases may be more cost effective. In situ studies evaluating mRNA expression will be needed to properly understand those cases which show moderate immunostaining (" $2+$ ") without genomic amplification. Reportedly, genomic amplification is usually associated with increased expression of messenger RNA and the encoded oncoprotein; discordance between expression and genomic amplification occurs in about 3 to $15 \%$ of cases. The clinical significance of lower levels of staining (" $2+$ ") and such discordance between genomic amplification and oncoprotein expression, including response to serotherapy, is uncertain.

Overall, the authors of the current study found very good concordance between genomic amplification and encoded protein detection by immunohistochemistry (19). Conclusions from other studies have not been as sanguine $(20,21)$. A particular problem has been an unexpectedly large number of positive results with immunohistochemistry using a polyclonal antibody approved by the FDA for determining Her-2/neu status. Surprisingly high percentages of Her-2/neu of positive cases were identified within a large series of breast carcinoma. When the same cases have been evaluated with a monoclonal antibody to the Her-2/neu encoded protein or with fluorescence in situ hybridization, 
the immunohistochemical results with the polyclonal antibody appear to be inappropriately high (21-23). Denying the potential clinical benefits of therapy must be carefully balanced against the significant cardiotoxicity associated with Herceptin administration. In particular, the prudent therapeutic choice for cases with " $2+$ " staining is uncertain.

The intense interest and controversy surrounding the laboratory detection of Her-2/ neu amplification is manifest in many ways. One need only look at the recently published abstracts from the annual United States and Canadian Academy of Pathology meeting to recognize the intense interest focused on this issue. FISH is inherently quantitative; results are expressed as average number of gene copies over multiple sampled fields. Yet FISH protocols are as variable as immunohistochemical methods, and special equipment is necessary. Because pretesting for technical and interpretative competence is required for both FISH FDA-approved systems before reagents can be purchased for clinical use, FISH variability is probably minimized as long as laboratories adhere to approve protocols. To our knowledge, such competence pretesting is not required for immunohistochemistry.

For immunohistochemistry, issues that have largely been ignored for some time are now forcibly brought to the fore for consideration. Should immunohistochemistry ever be considered a quantitative assay? Or are there at least limitations to be placed on quantitation of cytoplasmic membrane antigens (as opposed to nuclear determinants)? But even for relatively straightforward quantitation such as MIB/or Ki-67-based proliferation assays, numerous problems still remain if one considers intensity of the reaction as part of the quantitation. One could argue that immunohistochemistry has been developed as a qualitative adjunctive tool supplementing morphologic interpretation and was never really intended to be a quantitative technique. Certainly, given the enormous variability in procedural and interpretive protocols, intensity may simply be a variable that is unable to be clinically evaluated. In run cell-line quantitation control standards may improve reproducibility and reliability. Adherence to FDA-approved protocols for FDA-approved tests is an issue-deviation from such approved protocols, whether for "epitope retrieval," staining, or interpretation invalidates the FDA-approved status of the testing. The test becomes an in-house Analyte Specific Reagent (ASR)based test. Quality assurance programs administered by our profession's self-governing bodies still do not actively grade immunohistochemistry interlaboratory comparisons. One would think that in the third decade of use of diagnostic immunohistochemistry, a laboratory performing clinical assays should at least be able to consistently and reproducibly detect a core group of antigens such as cytokeratins, S100 protein, and basic lymphoid differentiation antigens. Yet there remains no graded rigorous quality assurance standard against which laboratories' results can be measured. The same can be said for interphase fluorescent and chromogenic in situ hybridization. More rigorous quality assurance programs for both immunohistochemistry and molecular morphology (FISH and chromogenic in situ hybridization) are absolutely essential.

Raymond R. Tubbs, D.O.
Department of Clinical Pathology
The Cleveland Clinic Foundation
Cleveland, Ohio
Mark H. Stoler, M.D.
Robert E. Fechner Laboratory of Surgical
Pathology
University of Virginia School of Medicine
Charlottesville, Virginia

\section{REFERENCES}

1. American Cancer Society. http://www.cancer.org/statistics/ cf99/selectedcancers.html\#breast. Accessed November 23, 1999.

2. Press MF, Bernstein L, Thomas PA, Meisner LF, Zhou JY, Ma $\mathrm{Y}$, et al. HER-2/neu gene amplification characterized by fluorescence in situ hybridization: poor prognosis in nodenegative breast carcinomas. J Clin Oncol 1997;15:2894-904.

3. Pauletti G, Godolpin W, Press MF, Slamon DJ. Detection and quantitation of HER-2/neu gene amplification in human breast cancer archival material using fluorescence in situ hybridization. Oncogene 1996;13:63-72.

4. Ro J, El-Naggar A, Ro J, et al. C-erb B-2 amplification in node-negative human breast cancer. Cancer Res 1989;49: 6941-4.

5. Seshadri R, Firgaria FA, Horsfall DJ, McCaul K, Setlur V, Kitchen P. Clinical significance of Her-2/neu oncogene amplification in primary breast cancer. J Clin Oncol 1993;11: 1936-42.

6. Slamon DJ, Godolphin W, Jones L, Holt J, Wong S, Keith D, et al. Studies of the Her-2/neu proto-oncogene in human breast and ovarian cancer. Science 1989;244:707-12.

7. Tsuda H, Hirohashi S, Shimosato Y, Hirota T, Tsugane S, Yamamoto $\mathrm{H}$, et al. Correlation between long-term survival in breast cancer patients and amplification of two putative oncogene coamplification units: hst-1 1/int- 2 and c-erb B-2/er-1. Cancer Res 1989;49:3104-8.

8. Kallioniemi OP, Holli K, Visakorpi T, Koivula T, Helin HH, Isola JJ. Association of c-erbB-2 protein over-expression with high rate of cell proliferation, increased risk of visceral metastasis and poor long-term survival in breast cancer. Int J Cancer 1991;49:650-5.

9. Babiak J, Hugh J, Poppema S. Significance of c-erbB-2 amplification and DNA aneuploidy. Analysis in 78 patients with node-negative breast cancer. Cancer 1992;70:770-6.

10. Toikkanen S, Helin H, Isola J, Joensuu H. Prognostic significance of HER-2 oncoprotein expression in breast cancer: a 30-year follow-up. J Clin Oncol 1992;10:1044-8.

11. Baselga J, Norton L, Albanell J, Kim YM, Mendelsohn J. Recombinant humanized anti-Her-2 antibody (Herceptin) enhances the antitumor activity of paclitaxel and doxorubi- 
cin against HER2/neu overexpressing human breast cancer xenografts. Cancer Res 1998;58:2825-31.

12. Baselga J, Tripathy D, Mendelsohn J, Baughman S, Benz CC, Dantis L, et al. Phase II study of weekly intravenous recombinant humanized anti-p185HER2 monoclonal antibody in patients with HER2/neu-overexpressing metastatic breast cancer. J Clin Oncol 1996;14:697-9.

13. Cobleigh MA, Vogle CL, Tripath D, Robert NJ, Scholl S, Fahrenbacher L. Efficacy and safety of Herceptin (humanized anti-HER2 antibody) as a single agent in 222 women with HER2 overexpression who relapsed following chemotherapy for metastatic breast cancer [abstract]. Proc Am Soc Clin Oncol 1998;17:97A;376.

14. Slamon D, Leyland-Jones B, Shak S. Addition of Herceptin (humanized anti-HER2 antibody) to first line chemotherapy for HER2 overexpressing metastatic breast cancer (HER2+ I MBC) markedly increases anticancer activity: a randomized, multinational controlled phase III trial [abstract]. Proc Am Soc Clin Oncol 1998;17:98A;377.

15. Thor AD, Berry DA, Budman DR, Muss HB, Kute T, Henderson IC, et al. erbB2, p53, and efficacy of adjuvant therapy in lymph node-positive breast cancer. J Natl Cancer Inst 1998; 90:1346-60.

16. Paik S, Bryant J, Park C, Fisher B, Tan-Chiu E, Hyams D, et al. erbB-2 and response to doxorubicin in patients with axillary lymph node-positive, hormone receptor-negative breast cancer. J Natl Cancer Inst 1998;90:1361-70.
17. Bianco AR, De Laurentiis M, Carlomagno C. 20-year update of the Naples GUN trial of adjuvant breast cancer therapy: evidence of interaction between c-erb-B2 expression and tamoxifen efficacy [abstract]. Proc Am Soc Clin Oncol 1998; 17:97A.

18. Gusterson BA, Gelbert RD, Goldhirsch A, Price KN, SaveSoderborgh J, Anbazhagan R, et al. Prognostic importance of c-erbB-2 expression in breast cancer. J Clin Oncol 1992;10: $1049-56$.

19. Jimenez RE, Wallis T, Tabasczka P, Visscher DW. Determination of Her-2/neu status in breast carcinoma: comparative analysis of immunohistochemistry and fluorescent in situ hybridization. Mod Pathol 2000;13:37-45.

20. Press MF, Hung G, Godolphin W, Slamon DJ. Sensitivity of HER-2/neu antibodies in archival tissue samples: potential source of error in immunohistochemical studies of oncogene expression. Cancer Res 1994;54:2771-7.

21. Roche PC, Ingle JN. Increased Her-2 with US Food and Drug Administration approved antibody. J Clin Oncol 1999;17: $434-5$.

22. Roche PC. CAP Today. June 1999. p. 16.

23. Jacobs TW, Gown AM, Yazijl H, Barnes MJ, Schmitt SJ. Specificity of HercepTest in determining Her-2/neu status of breast cancers using the United States Food and Drug Administration-Approved Scoring System. J Clin Oncol 1999; $17: 1983-7$. 